Non-axisymmetric self-similar flow between two rotating disks

Hewitt, R.E. and Al-Azhari, M.

2009

MIMS EPrint: 2012.20

Manchester Institute for Mathematical Sciences

School of Mathematics

The University of Manchester

\footnotetext{
Reports available from: http://eprints.maths.manchester.ac.uk/

And by contacting: The MIMS Secretary

School of Mathematics

The University of Manchester

Manchester, M13 9PL, UK
} 


\title{
Non-axisymmetric self-similar flow between two rotating disks
}

\author{
R. E. Hewitt • M. Al-Azhari
}

Received: 8 May 2007 / Accepted: 14 February 2008 / Published online: 7 March 2008

(C) Springer Science+Business Media B.V. 2008

\begin{abstract}
This paper considers the flow of an incompressible, viscous fluid forced by the independent rotation of two (bounding) infinite, parallel planes. The flow field is assumed to have a radial self-similarity of Von Kármán form and the relevant governing equations are derived with no assumptions of rotational symmetry. An exact class of solutions to the Navier-Stokes equations is shown to exist, corresponding to nonlinear, non-axisymmetric states. These steady, non-axisymmetric solutions appear through symmetry breaking of the classical axisymmetric steady states. The locus of bifurcation points is determined numerically and a number of limiting cases are described asymptotically. The initial-value problem is considered in the context of the self-similar equations. It is shown that unsteady calculations can break down at a finite time with the development of a singularity in the (exact) system of equations. An asymptotic description is given in the neighbourhood of the breakdown event. The structure of the singularity consists of an inviscid core flow to which an infinity of solutions are possible within the framework of the same asymptotic description. Whether a singularity is approached, or a steady/periodic axisymmetric state is achieved (and even the qualitative details of the singularity) is dependent on the initial conditions for some parameter regimes.
\end{abstract}

Keywords Finite-time singularity $\cdot$ Rotating disk $\cdot$ Symmetry breaking

\section{Introduction}

In this work we concentrate on a particular class of rotating-flow problem, namely the flow of a viscous fluid driven by the action of two independently rotating infinite, parallel planes. The problem of the flow between two rotating disks or above a single isolated disk has been a central problem in fluid dynamics for nearly 80 years. Von Kármán [1] first showed that, in the case of the flow above a single rotating disk, the full system of Navier-Stokes equations can be reduced exactly to a set of nonlinear, ordinary differential equations. This was achieved by assuming an axisymmetric, radial self-similarity to the form of solution.

The first accurate solution to Von Kármán's equations was presented by Cochran [2]. The disk was shown to act in the manner of a centrifugal fan, drawing fluid axially towards the disk surface and transporting it radially outwards. The contrary case to the Von Kármán solution is one in which the far-field fluid rotates at unit frequency

R. E. Hewitt $(\varangle) \cdot$ M. Al-Azhari

School of Mathematics, University of Manchester, Oxford Road, Manchester, England, UK

e-mail: richard.e.hewitt@manchester.ac.uk 
and the plane/disk is stationary. This case was later treated by Bödewadt [3] using the same self-similar solution structure proposed by Von Kármán.

The limiting cases presented by Von Kármán and Bödewadt were later generalized by Batchelor [4] to include the whole range of relative rotation rates; this formulation introduces a further parameter into the equations, namely the ratio of the angular frequencies of the far-field fluid and bounding surface.

Batchelor [4] and Stewartson [5] made further contributions to this area by extending the single-disk analysis to encompass the flow between two parallel disks that are allowed to rotate independently. The introduction of a lengthscale, in the form of the disk separation distance, introduces a Reynolds number $\left(\operatorname{Re}=\Omega_{0} L^{2} / \nu\right.$ ), which together with the relative angular frequencies of the disks $\left(s=\Omega_{1} / \Omega_{0}\right)$, forms a two-dimensional parameter space over which the solutions can be described. Here $L$ is the gap-width of the disks, $v$ is the kinematic viscosity of the fluid and $\Omega_{0}, \Omega_{1}$ the two angular frequencies of the bounding surfaces.

Batchelor argued that for high Reynolds numbers the flow should consist of a rigid-body core flow with a boundary layer at each disk. Stewartson presented an alternative view of the two-disk problem, suggesting that for the case of exact counter-rotation $(s=-1)$ or when one disk is fixed $(s=0)$, the circumferential motion would be confined to the boundary-layer regions. In fact, both types of solution are available to the two-disk problem and both have been observed experimentally; intriguingly, exactly which type of solution is found can depend crucially on the 'edge' (i.e., large radius) conditions (as suggested by Brady and Durlofsky [6]).

A large body of work has since been produced providing a detailed overview of the non-unique steady, radially self-similar, axisymmetric states available to the governing system of equations. In particular, Zandbergen and Dijkstra [7] provide an extensive survey of theoretical and numerical results concerning rotating-disk flows and discuss the relationship between one-disk and two-disk solutions.

Detailed numerical calculations have been provided by numerous authors; see, for example, [8] and [9]. These latter authors treat the parameter range of $s \in[-1,1]^{1}$ with a Reynolds number (as defined above) up to $10^{3}$. It was suggested that the axisymmetric solution structure is unique for Reynolds numbers in the range $\operatorname{Re} \lesssim 55$. Furthermore, as the Reynolds number is increased, the solution structure becomes increasingly complex, and as many as 19 steady states to the governing equations have been located in this regime.

Despite the large number of radially self-similar states, only a single bifurcation point (not of saddle-node type) was located, corresponding to the development of a new state at $\operatorname{Re} \approx 120, s=-1$. This bifurcation is related to a breaking of the reflectional mid-plane symmetry of the flow and has been classified as supercritical, with the symmetric state losing stability to the asymmetric state as the Reynolds number is increased. Many of the solution branches that have been located numerically, correspond to unusual (perhaps unphysical) velocity profiles, and a linear temporal stability analysis has suggested that, at sufficiently low Reynolds numbers, the unique state is stable to infinitesimal disturbances of the same radially self-similar form.

More recently, Hewitt and Duck [10] (subsequently referred to as HD) have shown that, in the case of a single rotating disk in an infinite fluid, there exist non-axisymmetric, stationary states that bifurcate from the axisymmetric solution branches at a number of critical ratios of the far-field and disk angular frequencies. HD noted that these states correspond to an exact reduction of the Navier-Stokes equations. It was also shown that the stationary states are in fact just one sub-class of a more general family of nonlinear, non-axisymmetric, travelling-wave solutions, although these more general solutions are not exact and rely upon a formal boundary-layer approximation. The exact, stationary, state described by HD was shown to appear subcritically and the analysis is therefore closely connected to the finite-amplitude instability described by Hall et al. [11].

The solution presented by HD for the boundary layer above a single rotating disk is unusual in that, although the radial and azimuthal velocity components are dependent on the azimuthal coordinate, the axial component remains axisymmetric. This feature suggests that a generalization may be expected in the form of analogous non-axisymmetric solutions to the flow between two parallel rotating disks, since at high Reynolds numbers a non-axisymmetric boundary layer at one of the disks can likely be matched to a similar structure at the other disk with an appropriate axial flow. It is this possibility that is investigated in this paper together with the corresponding initial-value problem.

1 This may be assumed without loss of generality since the equations can be rescaled if $s$ lies outside this region. 
The format of this paper is as follows. In Sect. 2 we derive the appropriate system of governing equations and show the form of the exact non-axisymmetric solution. Section 2.1 also provides details of the multiplicity of axisymmetric steady states available in the regime $s \in[-1,0)$. In Sect. 3 we locate (numerically) those bifurcation points in the range $\operatorname{Re} \in\left[0,10^{3}\right]$ which give rise to non-axisymmetric states. The numerical results prompt asymptotic investigations of the limiting cases of $\operatorname{Re} \rightarrow 0$ and $s \rightarrow 0^{-}$. Section 4 provides details of the corresponding initial-value problem for various parameter values and shows that evolutions that terminate in a breakdown can be achieved; an asymptotic description valid in the neighbourhood of the finite-time singularity is given. Finally, Sect. 5 provides some discussion and conclusions.

\section{Formulation}

We consider two parallel planes of infinite extent separated by a distance $L$ with the gap filled by a viscous fluid of kinematic viscosity $v$ and constant density $\rho$. The flow between the disks is described in terms of a cylindrical polar coordinate system $\left(r^{*}, z^{*}, \theta\right)$ centred at the axis of rotation of one of the disks. The disk at $z^{*}=0$ rotates with an angular frequency $\Omega_{0}$ and the disk at $z^{*}=L$ has an angular frequency of $\Omega_{1}$. We non-dimensionalize using the gap-width of the disks, $L$, as the natural lengthscale, $\Omega_{0} L$ as the velocity scale and $\Omega_{0}^{-1}$ as the timescale. Following the approach of von Kármán, we assume a radial self-similarity to the velocity field in the form

$\left(U^{*}, V^{*}, W^{*}\right)=\Omega_{0} L(r \hat{U}(z, \theta, t), \hat{V}(z, \theta, t), r \hat{W}(z, \theta, t))$,

where $r$ and $z$ are the non-dimensional coordinates defined by

$r^{*}=L r, \quad z^{*}=L z$

and $t^{*}=t \Omega_{0}^{-1}$. The pressure field is assumed to be in the form

$P^{*}=\rho\left(\Omega_{0} L\right)^{2}\left(\frac{r^{2} K}{2}+\hat{Q}(z, \theta, t)\right)$,

where $K$ is a constant.

Given this form of solution, we may reduce the Navier-Stokes equations to

$$
\begin{aligned}
& \hat{U}_{t}+\hat{U}^{2}+\hat{W} \hat{U}_{\theta}+\hat{V} \hat{U}_{z}-\hat{W}^{2}=-K+\frac{1}{\operatorname{Re}}\left(\hat{U}_{z z}+\frac{\hat{U}_{\theta \theta}-2 \hat{W}_{\theta}}{r^{2}}\right), \\
& \hat{W}_{t}+2 \hat{U} \hat{W}+\hat{V} \hat{W}_{z}+\hat{W} \hat{W}_{\theta}=-\frac{\hat{Q}_{\theta}}{r^{2}}+\frac{1}{\operatorname{Re}}\left(\hat{W}_{z z}+\frac{\hat{W}_{\theta \theta}+2 \hat{U}_{\theta}}{r^{2}}\right), \\
& \hat{V}_{t}+\hat{W} \hat{V}_{\theta}+\hat{V} \hat{V}_{z}=-\hat{Q}_{z}+\frac{1}{\operatorname{Re}}\left(\hat{V}_{z z}+\frac{\hat{V}_{\theta \theta}}{r^{2}}\right), \\
& 2 \hat{U}+\hat{V}_{z}+\hat{W}_{\theta}=0,
\end{aligned}
$$

where the subscripts denote differentiation and $\operatorname{Re}=\Omega_{0} L^{2} / \nu$. The appropriate boundary conditions are no-slip and impermeability of the two disks:

$\hat{U}(0, \theta, t)=\hat{V}(0, \theta, t)=0, \quad \hat{W}(0, \theta, t)=1$,

$\hat{U}(1, \theta, t)=\hat{V}(1, \theta, t)=0, \quad \hat{W}(1, \theta, t)=s$,

where $s=\Omega_{1} / \Omega_{0}$.

\subsection{A multiplicity of axisymmetric flow states}

For axisymmetric flows $(\partial / \partial \theta=0)$ an exact solution to system $(2)$ exists and is well known. The term "exact" here is used to indicate that there are no terms neglected in the reduced system of equations that one obtains. Here we 

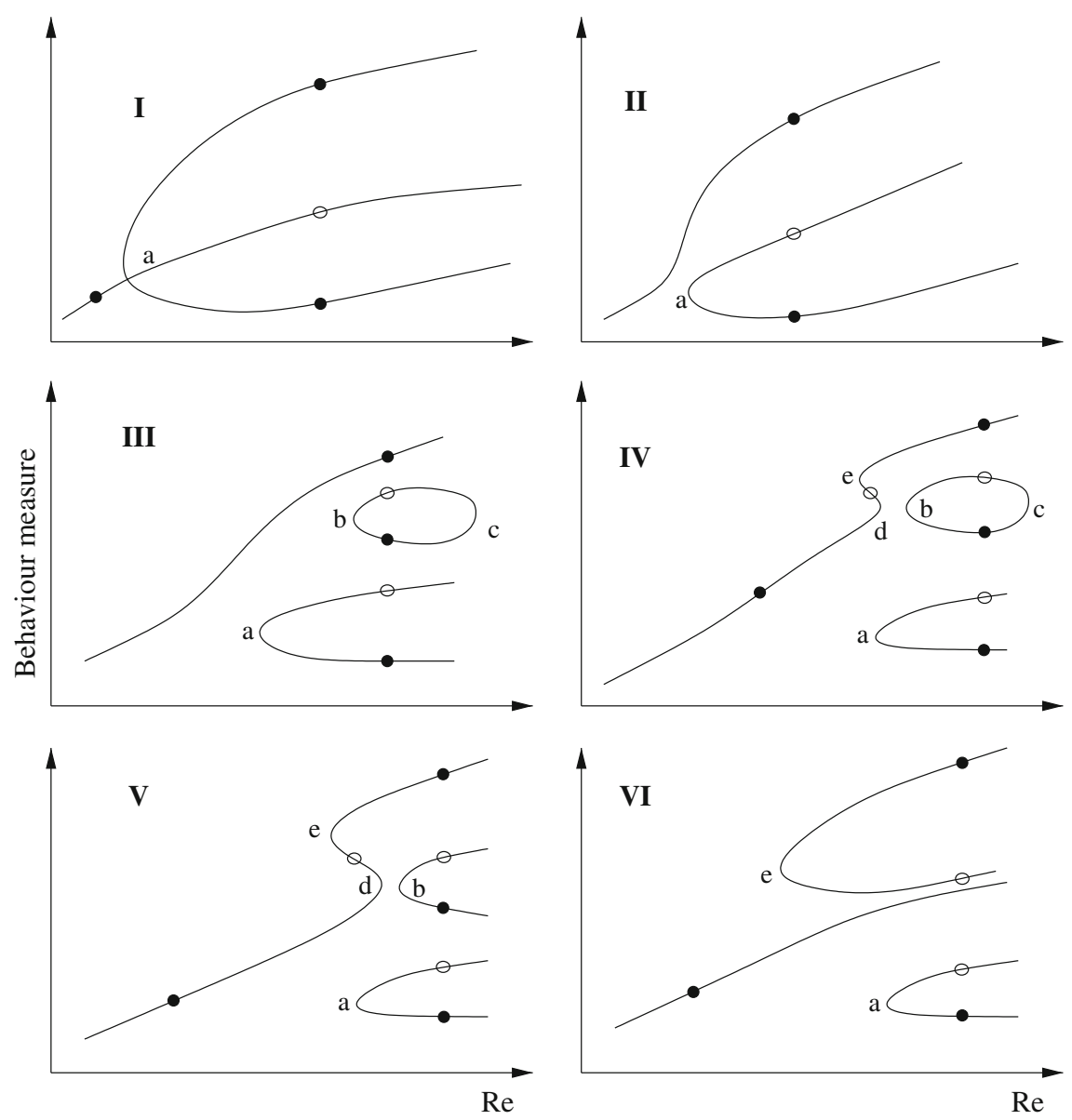

Fig. 1 A schematic of the axisymmetric steady solution structure at fixed values of the rotation ratio $s$. The labels (a)-(e) denote the fold points illustrated in Fig. 2 and the scenarios (I)-(VI) represent typical values of $s \in[-1,0)$, as given in Fig. 2. The open/filled circles denote unstable/stable solutions in the sense that these states can be reached by an axisymmetric initial-value calculation. Hopf bifurcations occur at higher Reynolds number and are not shown here

shall concentrate primarily on the case of counter-rotating disks, $s \in[-1,0)$, since it is in this regime that the main features of this work are most prominent. Some comments on the corresponding case of co-rotation, $s \in(0,1]$ will be given in the Sect. 4 .

A numerical procedure is used to compute the steady axisymmetric solutions of the governing system, which together with pseudo arc-length continuation allows a picture of the multiple states to be constructed. It is well recognized that the number of steady solutions increases with the Reynolds number, and here we present details only for Reynolds numbers in the range $\operatorname{Re} \in[0,1000]$, clarifying the structure of the solutions that play the dominant roles in affecting the unsteady response.

In Fig. 1 we illustrate how the sequence of bifurcations affects the solution structure for values of the rotation ratio, $s$, increasing from $s=-1$ in Fig. 1(I) to near zero in Fig. 1(VI). There is a supercritical bifurcation near $\operatorname{Re}=120$ at $s=-1$, which leads to the appearance to two solutions that break the reflectional mid-plane symmetry. For $s \neq-1$ this bifurcation is obviously structurally unstable, giving rise to the fold point (a). At higher values of $s$ an isola occurs over a fixed range of Re with two associated fold points (b) and (c).

As $s$ is increased we eventually encounter a cusp in the parameter space, giving rise to hysteretic behaviour and the fold points denoted by (d) and (e) in Fig. 1. At higher values of $s$ the limit point (c), denoting the fold of the isolated branch of states, tends to higher values of Re. 
On increasing $s$ yet further, a transcritical bifurcation is encountered as the fold points (d) and (b) merge, reconnecting the available flow states in a clearly different manner. Throughout this procedure, the fold point (a) persists and appears at higher Reynolds number.

We also note that supercritical Hopf bifurcations may also be located in this system. For example, the two stable solution branches that arise from the symmetry breaking bifurcation shown in Fig. 1 eventually lose stability and give rise to axisymmetric periodic states. Clearly, the Hopf bifurcation at $s=-1$ can be located on both branches of the solutions of Fig. 1(I) since they are merely reflections of each other in the mid-plane of the flow. These Hopf bifurcations persist for the full range of $s$ illustrated by Fig. 1. However, the appearance of these periodic states occurs only at much higher Reynolds number than we are concerned with in this work. In particular, for $s=-1$ the two Hopf bifurcations are located at $\operatorname{Re} \approx 2660$.

The definition of stable and unstable in the context of Fig. 1 (as denoted by the filled/open circles) is based on a linear stability analysis for axisymmetric perturbations. As we shall see, in the broader class of non-axisymmetric states that we describe in the next section these stability classifications can be altered. Furthermore, in the context of the initial-value problem to be considered in Sect.3, we shall see that each of the three stable states shown in Fig. 1 can be reached by time-marching the governing equations from three classes of initial condition, provided that the axisymmetry remains unbroken.

\subsection{Exact non-axisymmetric flow states}

To construct an exact, self-similar solution to system (2) one must consider only axisymmetric states or, alternatively, non-axisymmetric states that satisfy

$\hat{Q}_{\theta}=\hat{V}_{\theta}=\hat{U}_{\theta \theta}-2 \hat{W}_{\theta}=\hat{W}_{\theta \theta}+2 \hat{U}_{\theta}=0$;

thereby removing terms that would otherwise destroy the radial self similarity. Therefore, a solution to the system exists in the form

$$
\begin{aligned}
\hat{U}(z, \theta, t) & =U(z, t)+\Phi(z, t) \cos (2 \theta+\Delta), \\
\hat{V}(z, \theta, t) & =V(z, t), \\
\hat{W}(z, \theta, t) & =W(z, t)-\Phi(z, t) \sin (2 \theta+\Delta),
\end{aligned}
$$

for which it is self-consistent to take $\hat{Q}$ as a function of the axial coordinate, $z$, and time only. Here $\Delta$ is an arbitrary phase constant that we shall subsequently take to be zero without any loss of generality.

The resulting system of equations for this class of non-axisymmetric solutions is therefore

$U_{t}+U^{2}+V U_{z}+\Phi^{2}-W^{2}=\operatorname{Re}^{-1} U_{z z}-K, \quad W_{t}+2 U W+V W_{z}=\mathrm{Re}^{-1} W_{z z}$,

$2 U+V_{z}=0, \quad \Phi_{t}+2 U \Phi+V \Phi_{z}=\operatorname{Re}^{-1} \Phi_{z z}$,

together with the boundary conditions

$U(0, t)=V(0, t)=\Phi(0, t)=U(1, t)=V(1, t)=\Phi(1, t)=0$,

and

$W(0, t)=1, \quad W(1, t)=s$.

The unsteady problem can be closed by the formulation of appropriate initial conditions, details of which are provided in Sect. 4.

Hewitt and Duck [10] have shown that steady solutions of this special form exist in the case of a single rotating disk in a semi-infinite body of fluid; the appropriate parameter in their case is $W_{e}=\Omega_{\infty} / \Omega_{\text {disk }}$. The non-axisymmetric state arises subcritically at $W_{e} \approx-0.14485$ and continues nonlinearly to $W_{e}=0^{-}$at which point a breakdown of the steady solution structure is found involving a growing lengthscale. Prompted by the one-disk results, here we continue by seeking points in the two-dimensional parameter space at which a symmetry breaking bifurcation gives rise to non-axisymmetric steady states. The locus of these points in the $s-$ Re plane will be used to choose suitable parameter values for a subsequent discussion of the initial-value problem. 


\section{Bifurcations to steady, non-axisymmetric states}

Bifurcations to steady states for which

$\|\Phi(z)\| \equiv \sqrt{\int_{0}^{1}|\Phi(z)|^{2} \mathrm{~d} z} \neq 0$

(that is, non-axisymmetric states) are easily located in the standard manner. If $\|\Phi(z)\| \ll 1$, the coupling term $\Phi^{2}$ in (5a) can be neglected, and the leading-order equations are the classical, steady, rotating-disk equations combined with the steady form of (5d). A bifurcation point is then found at those steady axisymmetric states for which $U$ and $V$ are such that a steady solution to (5d) can be found that satisfies $\Phi(0)=\Phi(1)=0$. Further details of this procedure are given in the appendix to the paper by Hewitt et al. [12].

In general, these bifurcation points will form a path in the $s-$ Re parameter space. Furthermore, a weakly nonlinear description of the bifurcated non-axisymmetric state can be obtained in the usual manner (see Stuart [13]) by introducing an expansion valid near the bifurcation point. In this manner a Stuart-Landau type equation can be developed for the amplitude of the $\Phi$ component. We do not present such a description here since such an approach is entirely standard and numerical results for nonlinear states are easily obtained.

In Fig. 3 we show the locus of bifurcation points in the $s$-Re parameter space. These points are determined in the manner described above, and are associated with the primary axisymmetric state that extends from the limit of low Reynolds number. It can be seen from the figure that, as the Reynolds number is increased, two of the bifurcation points approach $s=0^{-}$while the other approaches a constant $s \approx-0.088$.

The bifurcation near $s \approx-0.088$ is easily described in terms of the previous analysis presented for the corresponding case of a single rotating disk. HD showed that a bifurcation to non-axisymmetric states can be found for a single disk when the ratio of the angular frequencies of the disk and far-field fluid is $\Omega_{\infty} / \Omega_{\text {disk }} \approx-0.1449$. Obviously, at sufficiently large Reynolds numbers, if the axisymmetric flow in the two-disk problem resembles a core-flow with two 'boundary layers' at each disk (i.e., it is of Batchelor type), then a bifurcation is obtained when $\Omega_{\text {core }} / \Omega_{\text {disk }} \approx-0.1449$. Such a state exists in the high Reynolds number limit for the two-disk geometry when $s \approx-0.088$.

We note that a temporal, linear, stability analysis of the steady state that extends from zero Reynolds number shows that the bifurcation points displayed in Fig. 3 are subcritical. Therefore, for a fixed $s$, we expect a threshold

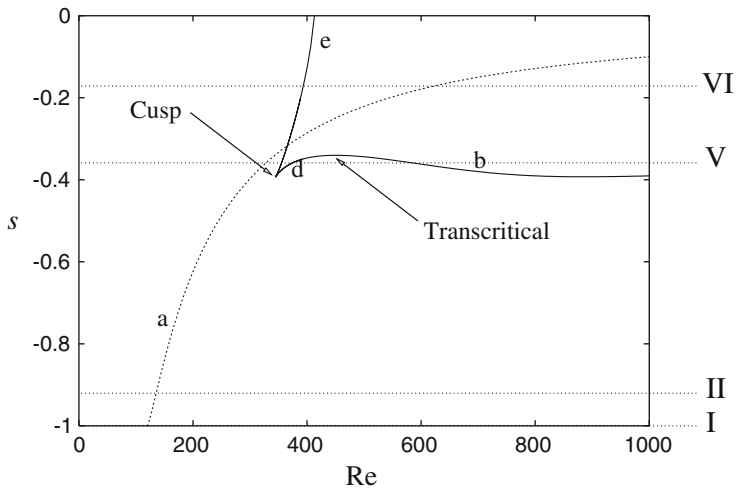

Fig. 2 Projections of the folds in the solution surface (as shown in Fig. 1) onto the parameter plane $s-\operatorname{Re}$. This data is obtained numerically from solution of the steady equations using continuation methods. The labels (a)-(e) correspond to those shown in Fig. 1. Note that the scenarios denoted by (III) and (IV) in Fig. 1 occur over a very narrow range of the parameter $s$ around the cusped region and are not shown here

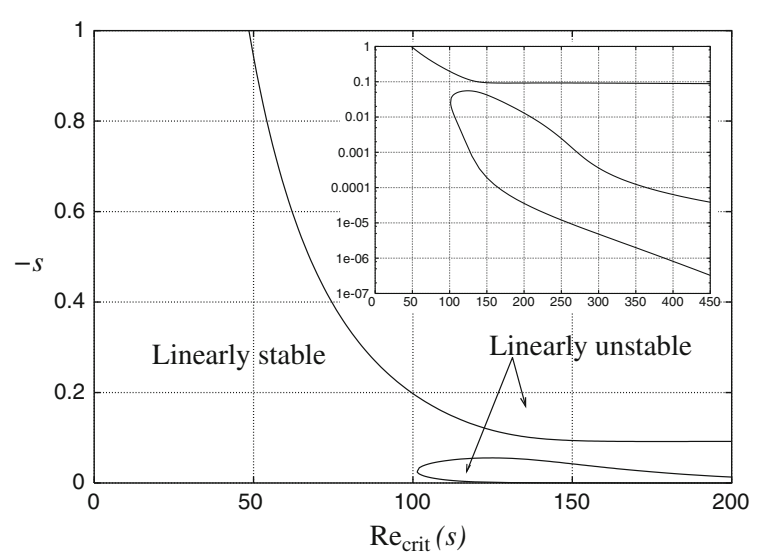

Fig. 3 Locus in the $s-$ Re plane of the (axial) symmetry breaking bifurcation, at $\operatorname{Re}=\operatorname{Re}_{\text {crit }}(s)$, from the primary axisymmetric solution that extend from the low Reynolds number limit. The inset figure shows the behaviour of the bifurcation points near to $s=0$ 
amplitude response for Reynolds numbers below the critical value $\operatorname{Re}_{\text {crit }}(s)$ and a linear instability for Reynolds numbers larger than this critical value.

\subsection{Numerically determined states}

In Fig. 4 we show a measure of the amplitude (the $L_{2}$-norm) of the non-axisymmetric component, $\Phi$, as the Reynolds number is increased in the case of exact counter-rotation $(s=-1)$. The (axial) symmetry breaking bifurcation is clearly visible, with the nonlinear, non-axisymmetric state arising near $\operatorname{Re} \approx 48.55$ and continuing nonlinearly as $\operatorname{Re} \rightarrow 0$. A further bifurcation is located at $\operatorname{Re} \approx 119.8$; however, the bifurcated state breaks the reflectional mid-plane symmetry in this case, rather than the axial symmetry.

In Fig. 6 we show the nonlinear states arising from the critical points illustrated in Fig. 3 for cross-sections of the parameter space at $\operatorname{Re}=75, \operatorname{Re}=95$ and $\operatorname{Re}=105$. It is readily observed from the figure that these states cannot be continued into the co-rotating ( $s>0$ ) half of the $s-\operatorname{Re}$ parameter space.

\subsection{The limit $\operatorname{Re} \rightarrow 0$ with $s=-1$}

It is clear from the form of Fig. 4 that, in this formulation, the nonlinear bifurcated state is singular as $\operatorname{Re} \rightarrow 0$. In this limit, an asymptotic description of the non-axisymmetric states can be developed in the form:

$(U(z), V(z), \Phi(z))=\left(U_{0}(z), V_{0}(z), \Phi_{0}(z)\right) \operatorname{Re}^{-1}+\cdots$,

$W(z)=W_{0}(z)+\cdots, \quad K=K_{0} \operatorname{Re}^{-2}+\cdots$.

The leading-order equations as $\mathrm{Re} \rightarrow 0$ are therefore

$U_{0}^{2}+V_{0} U_{0}^{\prime}+\Phi_{0}^{2}=U_{0}^{\prime \prime}-K_{0}, \quad 2 U_{0} W_{0}+V_{0} W_{0}^{\prime}=W_{0}^{\prime \prime}$,

$2 U_{0}+V_{0}^{\prime}=0, \quad 2 U_{0} \Phi_{0}+V_{0} \Phi_{0}^{\prime}=\Phi_{0}^{\prime \prime}$,

with boundary conditions

$U_{0}=V_{0}=\Phi_{0}=0, \quad$ on $z=0,1$,

and

$W_{0}(0)=1, \quad W_{0}(1)=s=-1$.

Solution of the system (8) is relatively straightforward, with the component $W_{0}(z)$ decoupled from the remaining three equations. However, it must be noted from the form of the equations satisfied by $W_{0}$ and $\Phi_{0}$, that a general solution can be given in the form

$W_{0}(z)=W_{0}^{*}(z)+A \Phi_{0}(z)$,

where $W_{0}^{*}$ is antisymmetric about $z=1 / 2$ and satisfies $W_{0}^{*}(0)=1$, and $W_{0}^{*}(1)=-1$. Here $A$ is an amplitude constant that remains undetermined at leading order. However, it can be shown that $A=0$ for this case of $s=-1$. Nevertheless, the introduction of an asymmetry at higher order can allow for a non-zero amplitude $A$ and it is in this manner that the expansions in the more general case may be connected to this description near $s=-1$.

3.3 The limit $\operatorname{Re} \rightarrow 0$ with $s=-1+\gamma \operatorname{Re}, \gamma=O(1)$

In this intermediate case the scaling for the axisymmetric azimuthal component is altered by the introduction of a small asymmetry proportional to the Reynolds number. The appropriate expansions are

$$
\begin{aligned}
& (U(z), V(z), W(z), \Phi(z))=\left(U_{0}(z), V_{0}(z), W_{0}(z), \Phi_{0}(z)\right) \operatorname{Re}^{-1} \\
& +\left(U_{1}(z), V_{1}(z), W_{1}(z), \Phi_{1}(z)\right)+\left(U_{2}(z), V_{2}(z), W_{2}(z), \Phi_{2}(z)\right) \operatorname{Re}+\cdots, \\
& K=K_{0} \operatorname{Re}^{-2}+K_{1} \operatorname{Re}^{-1}+K_{2}+\cdots,
\end{aligned}
$$


and the leading-order system in this case reduces to

$U_{0}^{2}+V_{0} U_{0}^{\prime}+\Phi_{0}^{2}-W_{0}^{2}=U_{0}^{\prime \prime}-K_{0}$,

together with $(8 \mathrm{~b})-(8 \mathrm{c})$ and $W_{0}(0)=W_{0}(1)=0$. Note that we have maintained the same zero index for the leading-order terms despite the different scaling for $W$ in this regime.

A solution can be sought in the form

$W_{0}(z)=A F_{0}(z), \quad \Phi_{0}(z)=B F_{0}(z)$,

where

$2 U_{0} F_{0}+V_{0} F_{0}^{\prime}=F_{0}^{\prime \prime}, \quad U_{0}^{2}+V_{0} U_{0}^{\prime}+F_{0}^{2}=U_{0}^{\prime \prime}-K_{0}, \quad 2 U_{0}+V_{0}^{\prime}=0$.

Here we have introduced two amplitude constants $A$ and $B$ under the assumption that

$B^{2}-A^{2}=1$.

In fact, (12a)-(12e) is a sixth-order system with the four no-slip boundary conditions on $U_{0}, \quad F_{0}$ and two impermeability conditions on $V_{0}$. Hence, the amplitude of $F_{0}(z)$ (i.e., $\left.F_{0}^{\prime}(0)\right)$ is fixed at leading-order but the two constants $A$ and $B$ remain undetermined at this stage, but can be computed at the order in which the asymmetry in boundary conditions is first introduced.

At next order in the expansion, the amplitudes $A$ and $B$ remain undetermined, with a general solution existing in the form

$\left(U_{1}, V_{1}, K_{1}\right)=A\left(U_{1}^{*}, V_{1}^{*}, K_{1}^{*}\right), \quad W_{1}=W_{1}^{*}+A^{2} F_{1}, \quad \Phi_{1}=A B F_{1}$,

where $U_{1}^{*}=V_{1}^{*}=F_{1}=0$ on $z=0,1$ and $W_{1}^{*}(0)=1, W_{1}^{*}(1)=-1$.

At the following order the amplitude term $A$ (and therefore $B$ ) are determined by a solvability condition applied to the resulting system. The solvability constraint determines the amplitude constant $A$ to be

$A(\gamma) \approx 9.167 \gamma$.

Thus for $\gamma=0$ we match this intermediate description with that provided in Sect. 3.2 and when $\gamma=O\left(\operatorname{Re}^{-1}\right)$ to the more general case discussed in the next section.

3.4 The limit $\mathrm{Re} \rightarrow 0$, at general $s$

Examination of the intermediate case of $\operatorname{Re} \rightarrow 0$ with $s=-1+\gamma \operatorname{Re}$, suggests that the amplitude of the leadingorder terms in the expansions for $W$ and $\Phi$ grow linearly with $\gamma$. Therefore, the low Reynolds number limit in the general case proceeds as above but with the replacement expansions

$W(z)=W_{0}(z) \operatorname{Re}^{-2}+\cdots, \quad \Phi(z)=\Phi_{0}(z) \operatorname{Re}^{-2}+\cdots$.

We can therefore derive a leading-order system of equations as $\mathrm{Re} \rightarrow 0$ in the form

$\Phi_{0}^{2}-W_{0}^{2}=0$,

together with (8b)-(8d). The appropriate boundary conditions are $U_{0}=V_{0}=W_{0}=\Phi_{0}=0$ at $z=0,1$.

Equation 19, together with (8b)-(8d), cannot be solved in isolation since insufficient information is given for the leading-order velocity component $U_{0}$. We can say however, that a solution has the following feature

$W_{0}(z)= \pm \Phi_{0}(z)$.

To determine the profile $\Phi_{0}(z)$ one must continue to next order in the expansion thereby obtaining a closed system of equations. 
The correction terms in the expansion procedure are all $O\left(\mathrm{Re}^{2}\right)$ smaller than the leading-order components and satisfy

$$
\begin{aligned}
U_{0}^{2}+V_{0} U_{0}^{\prime}+2 \Phi_{1} \Phi_{0}-2 W_{1} W_{0} & =U_{0}^{\prime \prime}-K_{0}, \\
2\left(U_{0} W_{1}+U_{1} W_{0}\right)+V_{0} W_{1}^{\prime}+V_{1} W_{0}^{\prime} & =W_{1}^{\prime \prime}, \\
2\left(U_{0} \Phi_{1}+U_{1} \Phi_{0}\right)+V_{0} \Phi_{1}^{\prime}+V_{1} \Phi_{0}^{\prime} & =\Phi_{1}^{\prime \prime},
\end{aligned}
$$

where $U_{1}=V_{1}=\Phi_{1}=0$ on $z=0,1, W(0)=1$ and $W(1)=s$. In this system $K_{0}$ is the leading-order term in the expansion of the pressure constant $K$. It is clear that this system depends on the correction terms $U_{1}(z)$ and $V_{1}(z)$, however, as we shall show, a closed system can be obtained by eliminating these terms.

Given (20), we suppose that $W_{0}(z)=\Phi_{0}(z)$ (the other sign can be treated in an analogous manner), and introduce a new quantity

$\Psi_{1}(z)=\Phi_{1}(z)-W_{1}(z)$.

Combining Eqs. 21b and 21c allows for a closed system of the form

$U_{0}^{2}+V_{0} U_{0}^{\prime}+2 \Phi_{0} \Psi_{1}=U_{0}^{\prime \prime}-K_{0}, \quad 2 U_{0} \Phi_{0}+V_{0} \Phi_{0}^{\prime}=\Phi_{0}^{\prime \prime}$,

$2 U_{0} \Psi_{1}+V_{0} \Psi_{1}^{\prime}=\Psi_{1}^{\prime \prime}, \quad 2 U_{0}+V_{0}^{\prime}=0$,

with boundary conditions $U_{0}=V_{0}=\Phi_{0}=0$ on $z=0,1, \Psi_{1}(0)=-1$ and $\Psi_{1}(1)=-s$. To obtain these equations we have eliminated $U_{1}$ and $V_{1}$ by subtracting (21b) from (21c) then combined the resulting equations with those found at leading order.

Numerical solution of (22a)-(22e) is straightforward and good agreement with the $\operatorname{Re} \rightarrow 0$ limit of the full equations is obtained (see Fig. 5). Similar results can be obtained in the case, $W_{0}(z)=-\Phi_{0}(z)$ by making a change of sign in the definition of $\Psi_{1}$. Thus, the small-Reynolds-number structure can be determined for any $s$, which matches to the above detailed expansion in the special case of $s \rightarrow-1$ via the intermediate structure outlined in Sect.3.3.

\subsection{The limit $s \rightarrow 0^{-}$for $\operatorname{Re}=O(1)$}

We have shown numerically, as illustrated by Fig. 6, that the bifurcated non-axisymmetric states have a limiting structure as $s \rightarrow 0^{-}$; an asymptotic description of this limit can also be provided.

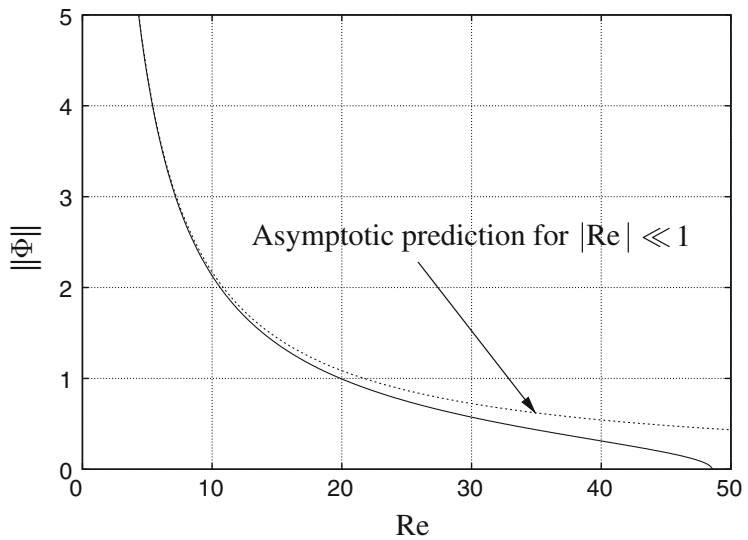

Fig. 4 Nonlinear continuation of the non-axisymmetric state arising from the bifurcation at $\operatorname{Re} \approx 48.55$ with $s=-1$. Here, we show the $L_{2}$-norm of the non-axisymmetric component. The dashed line indicates the asymptotic prediction of $\|\Phi\| \sim 21.75 / \mathrm{Re}$; as determined from Sect. 3.2

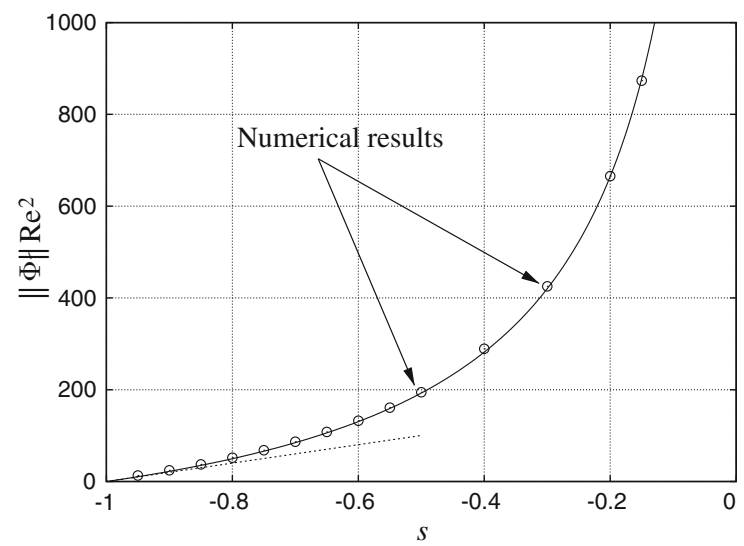

Fig. 5 A comparison of scaled numerical solutions (shown as circles) to the full system (5) (at $\operatorname{Re}=0.2$ ) with the $\|\operatorname{Re}\| \ll 1$ asymptotic prediction of 3.4 (the solid line). The scaling of the intermediate case discussed in Sect.3.2 is shown as the dashed line; this scaling predicts that $\|\Phi\| \operatorname{Re}^{2} \sim 21.75(1+$ $\left.9.17^{2}\right)^{1 / 2}(1+s)$ as $s \rightarrow-1$ 


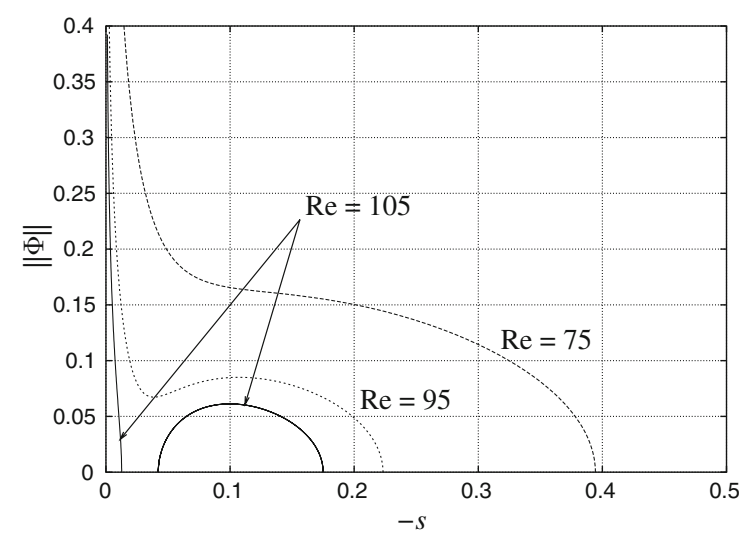

Fig. 6 Nonlinear non-axisymmetric states arising from the bifurcation points shown in Fig. 3 at fixed Reynolds number

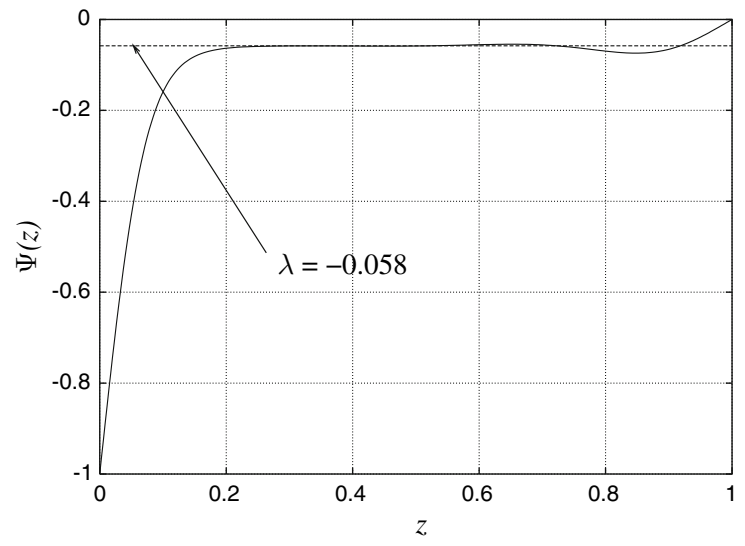

Fig. 7 A comparison of the leading-order core solution $\Psi_{0}(z)=\lambda$ with the full numerical $\Psi(z)$ as $s \rightarrow 0^{-}$with $\operatorname{Re}=1$. The comparison is made with $K \approx 10^{5}$ in the full solution

We shall assume $\operatorname{Re}=1$ (the only conceptual restriction is that $\operatorname{Re}=O(1)$ and we shall take it to be unity in order to simplify the notation). We also simplify the analysis by making the substitutions

$\Psi(z)=\Phi(z)-W(z)$,

and

$\Gamma(z)=\Phi(z)+W(z)$,

then the full governing system (5), with $\partial / \partial t=0$, may be written as

$U^{2}+V U^{\prime}+\Gamma \Psi=U^{\prime \prime}-K, \quad 2 U \Psi+V \Psi^{\prime}=\Psi^{\prime \prime}$,

$2 U \Gamma+V \Gamma^{\prime}=\Gamma^{\prime \prime}, \quad 2 U+V^{\prime}=0$,

with boundary conditions

$U=V=0, \quad \Psi=-1, \quad \Gamma=1, \quad$ on $z=0$,

$U=V=0, \quad \Psi=-s, \quad \Gamma=s, \quad$ on $z=1$.

The same analysis can be applied at more general Reynolds numbers with no qualitative changes, however we expect a "corner region" to exist for sufficiently small Reynolds numbers since there is a competing structure in this limit.

Numerical results for the full system (24) reveal that $K \rightarrow \infty$ as $s \rightarrow 0^{-}$in the continuation of the nonlinear, non-axisymmetric states. Rather than seeking an expansion in $|s|$ (small), we shall simply seek an expansion in terms of the pressure constant $K$ (large).

\subsubsection{The inviscid core: region II}

Numerical results suggest that a multi-layer structure is obtained, with an interior core region, $z=O(1)$, in which the appropriate scalings are

$\Psi(z)=\Psi_{0}(z)+\cdots, \quad \Gamma(z)=\Gamma_{0}(z) \epsilon^{-1}+\cdots, \quad V(z)=V_{0}(z) \epsilon^{-1 / 4}+\cdots, \quad U(z)=U_{0}(z)+\cdots$,

where $\epsilon=K^{-1}$.

The leading-order equations in the core are therefore

$\Psi_{0} \Gamma_{0}=-1, \quad V_{0} \Psi_{0}^{\prime}=0, \quad V_{0} \Gamma_{0}^{\prime}=0, \quad V_{0}^{\prime}=0$. 
which yield

$\Psi_{0}=\lambda, \quad \Gamma_{0}=-\lambda^{-1}, \quad V_{0}=V_{m}$,

where $\lambda$, and $V_{m}$ are constants to be determined.

We note that there are wall-layers in the flow on each disk; we refer to these as regions I and III near the lower disk and the upper disk, respectively.

\subsubsection{The viscous wall-layers: regions I and III}

Since $V, \Gamma$ and $\Psi$ in the interior are constant, their scalings in the layer I remain as given by (25), with leadingorder terms $\hat{V}_{0}(\zeta), \hat{\Gamma}_{0}(\zeta), \hat{\Psi}_{0}(\zeta)$. Furthermore, it is easy to note that, from a balance of the viscous terms, the appropriate wall-layer is defined in terms of a scaled co-ordinate of the form

$\zeta=\epsilon^{1 / 4} z=O(1)$

and therefore, from continuity,

$U(z)=\hat{U}_{0}(\zeta) \epsilon^{-1 / 2}+\cdots$.

The leading-order equations, to be satisfied in the wall-layer I, are therefore

$\hat{U}_{0}^{2}+\hat{V}_{0} \hat{U}_{0}^{\prime}+\hat{\Gamma}_{0} \hat{\Psi}_{0}=\hat{U}_{0}^{\prime \prime}-1, \quad 2 \hat{U}_{0} \hat{\Psi}_{0}+\hat{V}_{0} \hat{\Psi}_{0}^{\prime}=\hat{\Psi}_{0}^{\prime \prime}$,

$2 \hat{U}_{0} \hat{\Gamma}_{0}+\hat{V}_{0} \hat{\Gamma}_{0}^{\prime}=\hat{\Gamma}_{0}^{\prime \prime}, \quad 2 \hat{U}_{0}+\hat{V}_{0}^{\prime}=0$.

The boundary conditions to be satisfied are

$\hat{U}_{0}=\hat{V}_{0}=\hat{\Gamma}_{0}=0, \quad \hat{\Psi}_{0}=-1, \quad$ on $\zeta=0$,

$\hat{U}_{0}=0, \quad \hat{\Gamma}_{0}=-\lambda^{-1}, \quad \hat{\Psi}_{0}=\lambda \quad$ as $\zeta \rightarrow \infty$,

where $\lambda$ is a constant that remains to be determined.

The corresponding expansion in layer III is obviously of the same form, with $\hat{U}_{0}(\zeta), \hat{V}_{0}(\zeta), \hat{\Gamma}_{0}(\zeta)$ and $\hat{\Psi}_{0}(\zeta)$ replaced by $\tilde{U}_{0}(\xi),-\tilde{V}_{0}(\xi), \quad \tilde{\Gamma}_{0}(\xi)$ and $\tilde{\Psi}_{0}(\xi)$. Here the wall-layer is defined in terms of the coordinate $z=$ $1-\epsilon^{1 / 4} \xi$, where $\xi=O(1)$.

The resulting equations are then equivalent to (30) but with boundary conditions

$\tilde{U}_{0}=\tilde{V}_{0}=\tilde{\Gamma}_{0}=\tilde{\Psi}_{0}=0$, on $\xi=0$,

and

$\tilde{U}_{0}=0, \quad \tilde{\Gamma}_{0}=-\lambda^{-1}, \quad \tilde{\Psi}_{0}=\lambda \quad$ as $\quad \xi \rightarrow \infty$.

To determine $\lambda$, we must solve both systems for the regions I and III to determine the axial flow induced by each wall-layer as a function of $\lambda$. Since the core flow has a constant axial velocity, this must be matched to both wall-layer solutions. Therefore, the core flow is fixed by the value of $\lambda$ for which the axial flow in/out of layer I is the axial flow out/in to layer III, and it is this level of axial flow that exists uniformly across the inviscid interior. Therefore, we require that

$\hat{V}_{0}(\zeta \rightarrow \infty)=V_{m}=\tilde{V}_{0}(\xi \rightarrow \infty)$,

is satisfied.

Numerical solution of the governing equations in regions I and III, together with matching the Ekman mass transport of these layers across the inviscid core yields

$\lambda=-0.0580, \quad V_{m}=-1.349$.

In Fig. 7 we show a comparison of the flow component $\Psi(z)$ together with the predicted core value of $\lambda$ as $s \rightarrow 0^{-}$.

We must note that we have presented the asymptotic description in terms of $K \rightarrow \infty$ rather than in terms of a small parameter $|s|$. This description requires that

$s=g(K)$,

where $g(K)=o(1)$ in the limit $K \rightarrow \infty$. We note that the function $g(K)$ is only fixed at higher order in the expansion. Numerical evidence points to a logarithmic dependence of the form $K \sim(\log |s|)^{4}$, however we do not pursue this further here, having already clarified the leading-order structure. 


\section{The initial-value problem}

Here we wish to consider the corresponding initial-value problem for the full unsteady system defined by (5). We shall assume that the initial condition is one of rigid-body rotation at a constant rate $\Omega$ and with a possible small initial component that breaks the rotational symmetry, thus

$U(z, t=0)=V(z, t=0)=0, \quad W(z, t=0)=\Omega, \quad \Phi(z, t=0)=\delta \phi(z)$.

Here $\delta$ is an initial amplitude measure of the non-axisymmetric perturbation at $t=0$ and we shall typically take the initial profile to be $\phi(z)=z(1-z)$. The impact of the exact choice of initial profile, $\phi(z)$, on the evolution of the system will be discussed later. We note that the reflectional symmetry of $\phi(z)$ about the mid-plane $z=1 / 2$ may be anticipated to be important when $s=-1$, however, other initial perturbations have been tested that are not symmetric about $z=1 / 2$ with no qualitative differences observed.

In obtaining the computational results we assume that the disks change to their new rotation rates smoothly rather than impulsively; we choose to apply the conditions

$W(z=0, t \geq 0)=1+(\Omega-1) \mathrm{e}^{-c t^{2}}$,
$W(z=1, t \geq 0)=s+(\Omega-s) \mathrm{e}^{-c t^{2}}$,

where $c$ is a constant typically taken to be 10. Again, the exact form of change in rotation rate had no qualitative effect on the results that we present here. The purpose of (37) is to remove the singularity that would otherwise arise at $t=0$ if an impulsive transition was used.

In the preceding section we have presented details of those points in the two-dimensional parameter space at which bifurcations to non-axisymmetric states can be found. These non-axisymmetric branches arise from the steady, axisymmetric solution branch that exists for all Reynolds numbers. In all the cases discussed, the symmetry breaking bifurcations were found to be subcritical leading to a threshold amplitude response or linear instability of the axisymmetric state depending on the exact choice of parameters. The locus of the bifurcation points in the $s-$ Re parameter space obviously delineate regions in which the primary axisymmetric state is linearly unstable to non-axisymmetric perturbations of the form described here.

In the initial-value computations, three classes of evolution have been found. The system either approached an axisymmetric steady state, a periodic axisymmetric state or a (non-axisymmetric) finite-time singularity. In this work we provide just a few details regarding the axisymmetric states, whether stationary or periodic, and concentrate instead on the new feature introduced by allowing the flow to be non-axisymmetric, namely the finite-time singularity.

\subsection{Axisymmetric evolutions}

We begin by noting that there are clearly a number of states that are stable in the framework of the axisymmetric similarity equations, as illustrated in Fig. 1. Which of the multiplicity of axisymmetric steady states is achieved in the initial-value problem is dependent on the nature of the initial conditions.

Here we only consider initial conditions that are rigid-body rotations at some angular frequency $\Omega$ (i.e., $\delta=0$ ). For the parameter values $s=-0.3, \mathrm{Re}=900$, for example, we can achieve each of the three 'stable' (to infinitesimal axisymmetric self-similar perturbations) states shown in Fig. 1 (VI) by considering the cases $\Omega=0,1,-1$. These three initial conditions obviously correspond to introducing a slight counter-rotation from an initial state of: rest $(\Omega=0)$; rotation at the frequency of the faster (final) rate of the two disks $(\Omega=1)$; and rotation at this same frequency but in the opposite direction $(\Omega=-1)$.

In Fig. 8 we show three evolutions from the these three distinct initial conditions. The solutions are each characterized by the corresponding pressure constant $K$, and the three steady-state values of $K$ are also shown. 
4.2 Structure in the neighbourhood of the non-axisymmetric breakdown event

In Fig. 9, we show the time evolution of the shear components $U_{z}(z=0, t)$ and $U_{z}(z=1, t)$, providing numerical evidence for the presence of a singularity. Given the results of Fig. 9, we suppose that the governing system possesses a singularity at a finite, non-dimensional time of $t=t_{s}$. Therefore, we define a new variable

$\tau=t_{s}-t$

and seek an asymptotic description in the limit of $\tau \rightarrow 0$. The description in this limit takes on a multi-layered structure, which consists mainly of two viscous layers at each boundary and an inviscid core flow.

\subsubsection{The inviscid core}

If we suppose that there is an inviscid core region in which $z=O(1)$, then a balance of the acceleration, pressure and nonlinear terms, suggests an expansion of the form

$$
\begin{aligned}
(U(z, t), V(z, t), W(z, t), \Phi(z, t)) & =\left(u_{0}(z), v_{0}(z), w_{0}(z), \phi_{0}(z)\right) \tau^{-1}+\cdots, \\
K & =k_{0} \tau^{-2}+\cdots .
\end{aligned}
$$

The resulting leading-order equations in the core are therefore

$u_{0}+u_{0}^{2}+u_{0}^{\prime} v_{0}+\phi_{0}^{2}-w_{0}^{2}=-k_{0}, \quad 2 u_{0}+v_{0}^{\prime}=0$,
$\phi_{0}+2 u_{0} \phi_{0}+v_{0} \phi_{0}^{\prime}=0, \quad w_{0}+2 u_{0} w_{0}+v_{0} w_{0}^{\prime}=0$.

Since $\phi_{0}$ and $w_{0}$ satisfy the same linear equation, we make a simplifying substitution of the form

$\phi_{0}(z)=\bar{B} f_{0}(z), \quad w_{0}(z)=\bar{A} f_{0}(z)$,

where $\bar{A}$ and $\bar{B}$ are amplitude constants, which without loss of generality can be taken such that

$\bar{B}^{2}-\bar{A}^{2}=1$,

provided that we exclude the case $\bar{A}=\bar{B}$; since there is no numerical evidence for such a case. Here, $f_{0}(z)$ is a function that satisfies the same form of first-order equation as $\phi_{0}$ and $w_{0}$, but whose 'amplitude' is uniquely determined in this layer. We note that the constants $\bar{A}$ and $\bar{B}$ will remain undetermined.

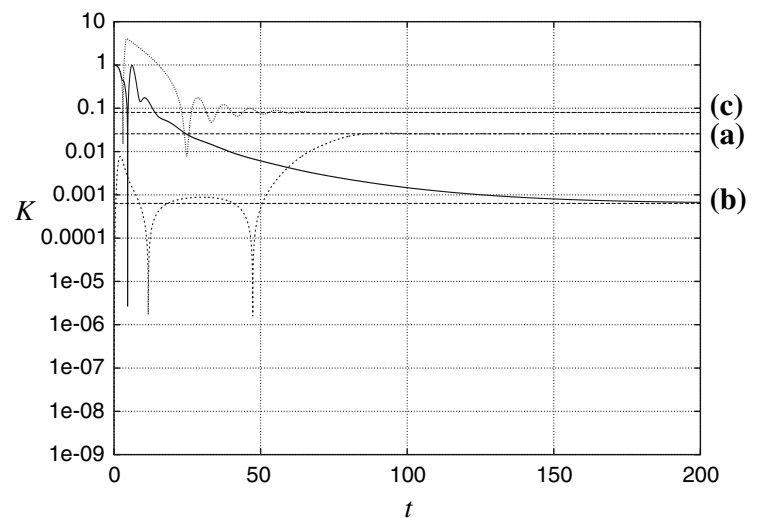

Fig. 8 Three axisymmetric evolutions starting from the three different initial conditions of (a) no motion $\Omega=0$, (b) rigidbody rotation at $\Omega=1$ and (c) rigid-body rotation at $\Omega=-1$. Here we show the variation of the pressure constant $K$ with time for the parameter values $s=-0.3$ and $\mathrm{Re}=900$. The corresponding steady-state values are shown as the horizontal dashed lines

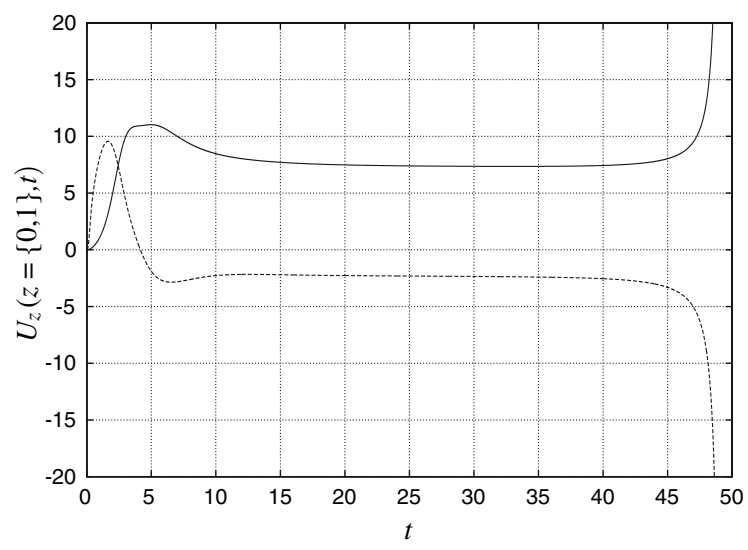

Fig. 9 The evolution of $U_{z}(z=0, t)$ (upper, solid, curve) and $U_{z}(z=1, t)$ (lower, dashed, curve) in the case $s=-1 / 2$ $(\operatorname{Re}=200)$, showing the evolution towards a singularity as $t \rightarrow t_{s} \approx 49$ 

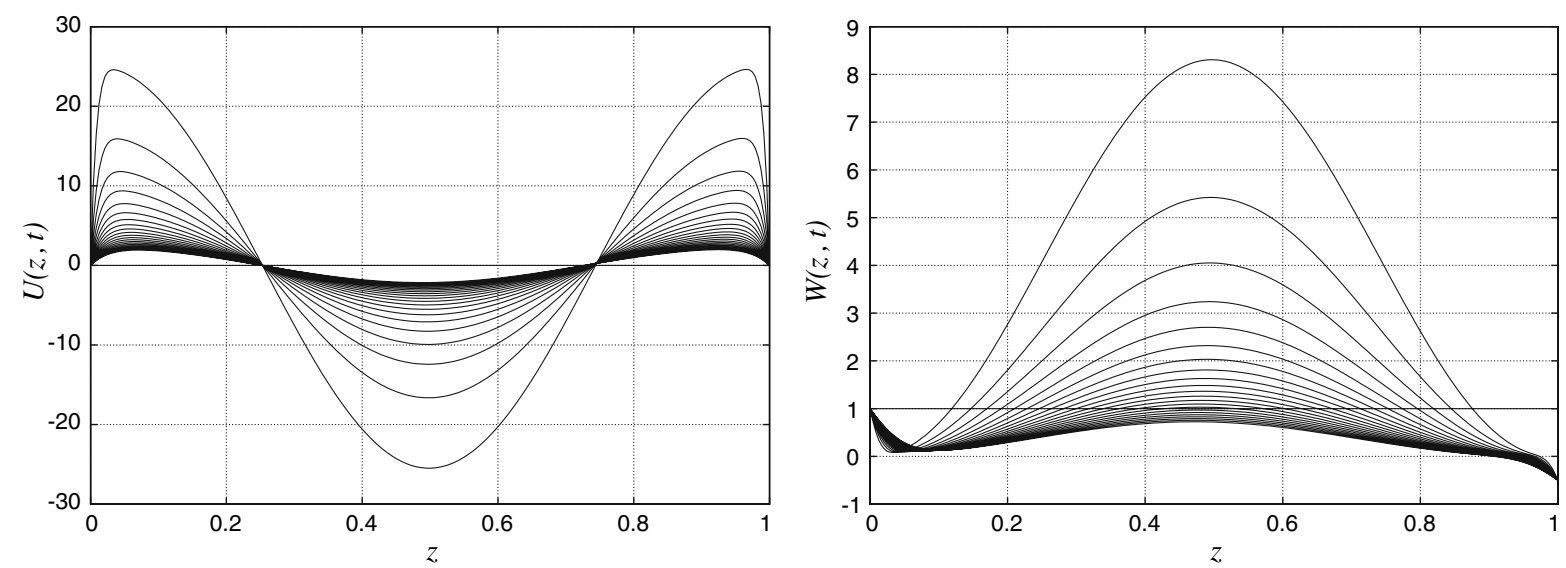

Fig. 10 Profiles of the velocity components $U$ and $W$ as $t \rightarrow t_{s}$ in the case of $s=-1 / 2$ with $\operatorname{Re}=200$. Profiles are shown at $t=49.01,49.02, \ldots, 49.22$

Prompted by the form of the profiles shown in Fig. 10, the behaviour of the solution on approaching the boundaries can be obtained by supposing that $u_{0}(z) \sim \beta$ as $z \rightarrow 0$ (or equivalently as $z \rightarrow 1$ ), where $\beta$ is a constant. Therefore, from (40a) we find that

$\beta^{2}+\beta+k_{0}=0$.

It is perhaps unsurprising that the system of equations (40) is somewhat similar in nature to the inviscid equations that arise in describing the class of singular, steady, solutions to the flow above a single rotating disk. In fact, (40) admits solutions akin to the 'inviscid cell' type states that exist in the classical rotating-disk equations.

We seek a general solution to the inviscid core equations in the form

$u_{0}(z)=\beta \cos [\mu z], \quad v_{0}(z)=-2 \beta \sin [\mu z] / \mu, \quad f_{0}(z)=\gamma(1-\cos [\mu z])$,

where $\mu, \beta$ and $\gamma$ are constants. Such solutions are easily shown to exist provided that

$\beta=1 / 2, \quad$ and $\gamma=1 / 2$.

Thus, the pressure constant is determined from (43) to be

$k_{0}=-3 / 4$.

We note at this stage that, if we choose $\mu=2 \pi$, then (44) provides an outer solution that satisfies impermeability at the two rigid boundaries of $z=0$ and $z=1$. This inviscid solution can then be matched with appropriate viscous regions to satisfy the no-slip boundary conditions. However, in general we can seek solutions with $\mu=2 m \pi$ and still match to wall layers at $z=0,1$.

\subsubsection{The viscous layers}

As can be observed from the form of (40) and (44) further viscous regions can exist in the limit of $z \rightarrow n \pi / \mu<1$ as the leading-order axial flow vanishes (here $n=1,2,3 \ldots$ ). On approaching these levels in the flow field, the behaviour of the leading-order velocity components is

$u_{0}=O(1), \quad v_{0}=O(z-n \pi / \mu)$,

$f_{0} \sim\left(1-(-1)^{n}+\mu^{2}(z-n \pi / \mu)^{2}(-1)^{n} / 2\right) / 2$.

Thus, for even values of the integer $n=2 m, f_{0}=O\left((z-n \pi / \mu)^{2}\right)$. 
The depth of the viscous layer is easily determined by a balance of the acceleration, viscous and pressure terms, and we can define a new coordinate that spans this region,

$z=2 m \pi / \mu+\tau^{1 / 2} \eta$.

The corresponding expansions are of the form

$U(z, t)=\tilde{u}_{0}(\eta) \tau^{-1}+\cdots, \quad V(z, t)=\tilde{v}_{0}(\eta) \tau^{-1 / 2}+\cdots, \quad(W(z, t), \Phi(z, t))=\left(\tilde{w}_{0}(\eta), \tilde{\phi}_{0}(\eta)\right)+\cdots$,

while the pressure constant is

$K=k_{0} \tau^{-2}+\cdots$,

as before.

The appropriate leading-order equations in this region are therefore

$\tilde{u}+\eta \tilde{u}_{0}^{\prime} / 2+\tilde{u}^{2}+\tilde{v} \tilde{u}^{\prime}=\tilde{u}^{\prime \prime}-k_{0}, \quad 2 \tilde{u}+\tilde{v}^{\prime}=0, \quad \eta \tilde{\phi}_{0} / 2+2 \tilde{u} \tilde{\phi}_{0}+\tilde{v} \tilde{\phi}_{0}^{\prime}=\tilde{\phi}^{\prime \prime}$,

together with the same equation with $\tilde{\phi}_{0}$ replaced by $\tilde{w}_{0}$. We note that the equations (51a) and (51b) are decoupled from the two additional equations for $\tilde{w}_{0}$ and $\tilde{\phi}_{0}$.

If $2 m \pi / \mu=0$ (or 1 ) then the appropriate boundary conditions at the disks are no-slip and impermeability, $\tilde{u}_{0}(0)=\tilde{v}_{0}(0)=\tilde{\phi}_{0}(0)=0, \quad \tilde{w}_{0}(0)=1$, with similar conditions at $z=1$, except that $\tilde{w}_{0}(1)=s$. In these regions the solution needs to be determined numerically and matched to the outer core solution.

If $2 m \pi / \mu$ is not zero or unity, then layer is in the central core flow. However, these interior layers are easily seen to be entirely passive and can be ignored.

Therefore, in general, the solution in the core has $\mu=2 m \pi(m=1,2,3 \ldots)$. When $m=1$ the solution matches directly to wall layers at $z=0,1$. For $m>1$, there are multiple inviscid cells in the core flow, with the first and last cell again matched to the viscous wall layers. It is clear that the temporal evolution shown in Fig. 10 corresponds to an interior solution with $m=1$.

We note that the small- $\tau$ asymptotics only determine the 'amplitude' of $f_{0}(z)$, that is, although we know the size of $\phi_{0}^{2}-w_{0}^{2}$ in the core region, we cannot determine the individual amplitude constants $\bar{B}$ and $\bar{A}$. In general, these quantities depend on an outer region as $\tau \rightarrow \infty$ (i.e., the history of the evolution).

\subsection{Comparison of asymptotic and numerical results}

In Fig. 11, we show the temporal evolution of the quantity $z_{\min }$, which is the $z$-location of the minimum value of the radial component $U(z, t)$ (as shown in Fig. 10). In this case, $s=-1 / 2, \mathrm{Re}=200$, and in agreement with the asymptotic description of the singularity, the core-flow becomes symmetric about the mid-plane with $z_{\min } \rightarrow 1 / 2$ as $\tau \rightarrow 0$.

In Fig. 12, we show a comparison between two scaled quantities evaluated from the full numerical solution as $t \rightarrow t_{s}$ and the appropriate asymptotic predictions. In particular, given the interior solution, we observe that the quantity $U_{\min }(t)=U\left(z=z_{\min }, t\right)$ should have a leading-order description

$U_{\min }=-(1 / 2) \tau^{-1}+\cdots$,

therefore $\left|U_{\min }\right|^{-1} \sim 2 \tau$ as $\tau \rightarrow 0$. Similarly, since

$K=-(3 / 4) \tau^{-2}+\cdots$,

we compare $|K|^{-1 / 2}$ with $(3 / 4)^{-1 / 2} \tau$.

A further prediction of the asymptotic description is that the inviscid interior region can have an infinity of solutions of the form

$U(z, t)=(1 / 2) \cos [2 m \pi z] \tau^{-1}+\cdots$, 


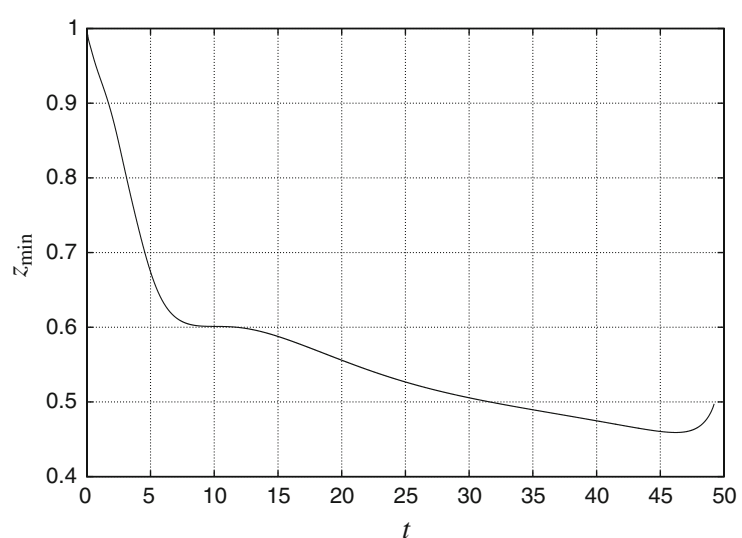

Fig. 11 The location of the central minimum in the profile $U . U_{\min }=U\left(z=z_{\min }, t\right)$ for $s=-1 / 2$ and $\operatorname{Re}=200$. Even though $s \neq-1$ the interior solution approaches a state that is symmetric about $z=1 / 2$ in the neighbourhood of the singularity

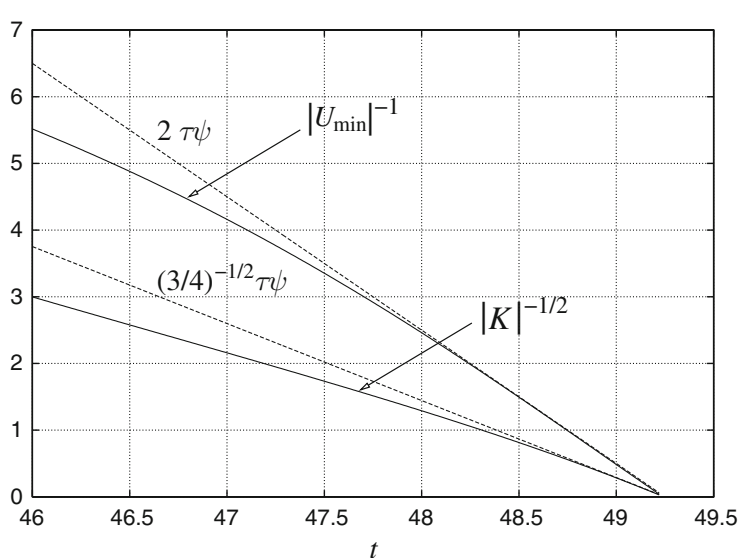

Fig. 12 Comparison of scaled values obtained from the full numerical solution (solid lines) in the neighbourhood of the breakdown event with the predictions of the asymptotic description (dashed lines) in the core region

for any $m=1,2, \ldots$. By choosing an initial condition with a larger initial amplitude $\delta$ and a functional form

$\phi(z)=1-\cos [4 \pi z]$,

an unsteady evolution to a singularity contained within the same asymptotic framework but with $m=2$ can be found, as shown in Fig. 13. Similar figures can be generated that correspond to core-flows with $m=3,4, \ldots$; however, the computations suggest that to trigger such evolutions requires large initial non-axisymmetric perturbations.

In general, if $\operatorname{Re}>\operatorname{Re}_{\text {crit }}(s)$ (as shown in Fig. 3), the linear instability of the axisymmetric state results in the initial-value problem eventually approaching a singularity (with $m=1$ ) for sufficiently small amplitudes, $\delta$, of the initial perturbation.

\subsection{More complex scenarios}

As we have observed, there are a number of steady axisymmetric states that are linearly stable in the framework of the similarity equations provided that the axisymmetry remains unbroken. However, in our analysis only the solution state that extends to low Reynolds numbers has been found to possess a region that is linearly stable to both axisymmetric and non-axisymmetric perturbations. That is, the disconnected states illustrated in Fig. 1 are found to be unstable to non-axisymmetric perturbations.

As can be seen from comparisons of Figs. 2 and 3, one can choose parameter values for $s$ and Re for which the primary branch of steady solutions (that extends from low Reynolds numbers) is stable to non-axisymmetric modes, but for which other states exist that are linearly stable to axisymmetric perturbations and unstable to non-axisymmetric perturbations. For example, the parameter values $s=-0.05, \operatorname{Re}=500$ are in the stable region of Fig. 3 , therefore the primary steady state is linearly stable to non-axisymmetric modes, but there are other states that are unstable to non-axisymmetric modes. In this context the evolution of the system can depend dramatically on the initial conditions.

For example, if we choose initial conditions of $\Omega=0$ and a small, $\delta$ (thereby breaking the rotational symmetry), then an evolution to the stable primary state is achieved. However, for an initial condition of $\Omega=-1(\delta \neq 0)$, the evolution begins to approach one of the alternative steady states, which though stable to axisymmetric perturbations are linearly unstable to modes that break the axisymmetry. In this latter case the evolution is towards a finite-time singularity of the form described above. 

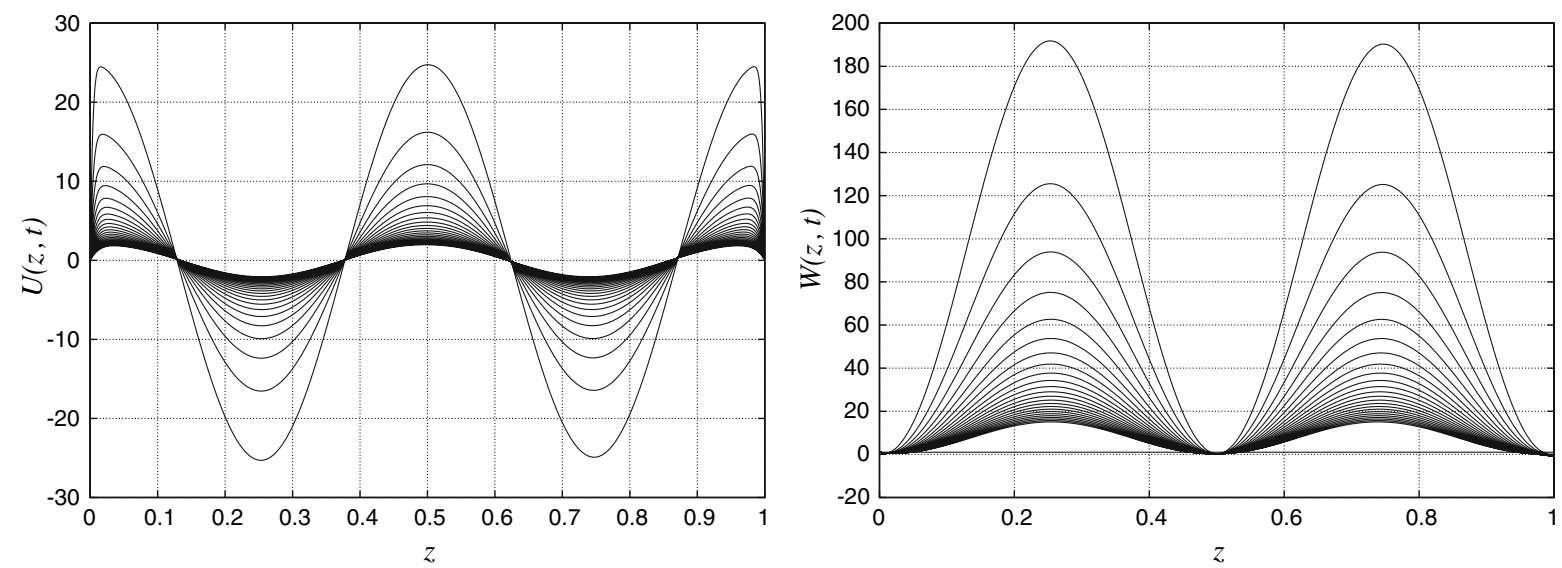

Fig. 13 Profiles of the velocity component $U$ and $W$ as $t \rightarrow t_{s}$ in the case of $s=-1$. In this instance a different core flow is obtained by altering the initial conditions; further details are given in Sect. 4.3. Compare this evolution with the $m=1$ case shown in Fig. 10

\section{Discussion}

We have considered an unsteady three-dimensional solution that results in an exact reduction of the Navier-Stokes equations to an eighth-order nonlinear system of ordinary differential equations. The solution corresponds to a class of non-axisymmetric flow, with an azimuthal wavenumber of two, confined between two, infinite, parallel, co-axial rotating disks.

Non-axisymmetric states have been shown to arise through bifurcations from the branch of axisymmetric states that develop from the Stokes flow limit. The bifurcations lead to steady, nonlinear states that terminate with a singular structure on approaching the critical boundaries of $\operatorname{Re}=0$ and $s=0$. Asymptotic descriptions of these limits have been presented. The bifurcations are seen to be subcritical in nature, typically leading to a threshold amplitude response at low Re for fixed $s<0$. However, there is a broad region of the $s<0$ part of parameter space for which a linear instability is predicted for modes that break the rotational symmetry. We should note that the singular behaviour as $\mathrm{Re} \rightarrow 0$ is indicating that a Reynolds number based on the rotation of the boundaries is not the most representative choice for these bifurcated states; i.e., that there is significant motion in the bulk fluid despite the fact that Re is small.

Unsteady computations relevant to the initial-value problem confirm the presence of both the threshold amplitude response and the linear instability. For states that are unstable to modes of the type described here, we have presented numerical results that suggest the system evolves to a finite-time singularity. An asymptotic description valid near the breakdown event has also been presented that compares well with the full numerical results. As we have noted, Hall et al. [11] have previously described a similar problem, showing a threshold amplitude response and eventual finite-time breakdown of Von Kármán's solution to the flow over a single rotating disk. The structure of the singularity described by Hall et al. is more complicated than that presented here, including as it does a 'boundary-layer' 2 thickness that increases without bound as the singularity is approached. Such a scenario is not possible for a flow confined by two disks, and the structure of the breakdown presented here must necessarily be somewhat different.

As the singularity is approached the solution consists of an inviscid core flow with two wall-layers of decreasing thickness at each boundary. When the flow evolves towards the singularity, the core flow becomes increasingly symmetric about the mid-plane $z=1 / 2$, even if the initial conditions or the boundary conditions contain no such symmetry. Furthermore, in the description of the breakdown, there is an infinity of solutions to the equations appropriate to the inviscid region, parameterized by an integer $m$. Which of the inviscid solutions is realized in the

\footnotetext{
${ }^{2}$ We still use the phrase 'boundary-layer' although we recognize that no associated approximation is necessary in this instance.
} 
unsteady calculations is dependent on the initial conditions. Typically, the flow will evolve to a breakdown with $m=1$, however finite-time singularities that correspond to different values of $m$ can be achieved by an appropriate choice of initial conditions. In general, the evolutions that break down with a core-flow that contains a 'higher- $m$ ' solution are more difficult to achieve and require successively larger initial perturbations; that is, they require a large $\delta$ in (36). There is no evidence of similar bifurcations from the primary branch of steady states being available in the parameter regime $s \geq 0$. However, the instability of the other solution states to non-axisymmetric perturbations persists and so evolutions to a breakdown at a finite time can still be achieved for some classes of initial condition as outlined in Sect. 4.4.

The question of the practical relevance of the finite-time singularity discussed herein is a subtle one. Because the solution provides an exact reduction of the Navier-Stokes equations (NSE) this case is somewhat different from those obtained, for example, in boundary-layer theory; for which a return to the full field equations is achieved on approaching the singularity. In this problem the singular solution structure is also clearly a solution of the full NSE. The most likely scenarios are therefore: (i) instabilities of a more general spatial nature must eventually dominate the time evolution of the system, thereby preventing the singularity formation and/or (ii) the finite-domain nature of any real flow leads to a spatio-temporal evolution that is distinct from this infinite-domain case. A fully unsteady, three-dimensional, finite-domain formulation and numerical solution would clearly be of interest in this respect (especially with regards comparisons in the near axis, small- $r$, region).

The previous analysis of HD extended the class of exact solution to more general azimuthal wavenumbers, whilst also allowing for travelling-wave states. However, we note that this approach is only valid following a formal boundary-layer approximation and only describes the flow sufficiently far from the rotation axis; that is $r \gg \operatorname{Re}^{-1 / 2}$. Without this approximation the $r^{-2}$ terms present in (2a)-(2d) cannot be neglected and a radially self-similar solution cannot be applied. Therefore, for the flow between two disks at finite Reynolds numbers, one cannot seek to determine the stability of the flow to more general forms of azimuthally propagating waves without relaxing the self-similar form of the perturbation. Nevertheless, at sufficiently large Reynolds numbers, the analysis of HD can apply locally near to each of the disks provided that the axisymmetric solution consists of two boundary layers and a core-flow that is a rigid body rotation.

Our primary motivation for this work was to describe a new exact solution of the Navier-Stokes equations; because such solutions are rare, they are generally always of some interest. However our long-term aim is to develop similarity solutions that display non-uniqueness and bifurcation phenomena as a basis to investigate the significance of such simplified solutions to large but finite aspect-ratio problems. In this regard, the recent work of Hewitt and Hazel [14] has investigated the relevance of the axisymmetric solutions to (2) by numerical computation of the full axisymmetric field equations in a finite geometry with a variety of end conditions applied at the edge of the disks. The Von Kármán-type similarity solutions are often assumed to apply in a 'sufficiently large' domain, however the results of Hewitt and Hazel show that this assumption is often false. We make no claims that the special form of exact solution that we describe will be dominant in any experimental configuration, the question of the role of these exact solutions in large but finite domains is a subtle one, particularly in the context of stability theory. Indeed the very recent results of Nore et al. [15] point to time-dependent axisymmetric instabilities being the dominant instability in large-aspect-ratio counter-rotating-disk flows within a closed surrounding cylinder. The role of the non-axisymmetric mode described herein (in the context of the global eigenvalue problem) is worthy of further investigation, particularly in regard to sensitivity to conditions imposed at the edge of the domain.

\section{References}

1. von Kármán T (1921) Über laminare und turbulente Reibung. ZAMP 1:233-252

2. Cochran WG (1934) The flow due to a rotating disk. Cam Phil Soc 30:365-375

3. Bödewadt UT (1940) Die Drehströmung Über festem Grund. ZAMM 20:241-245

4. Batchelor GK (1951) Note on a class of solutions of the Navier-Stokes equations representing steady rotationally-symmetric flow. Quart J Mech Appl Math 4:29-41

5. Stewartson K (1953) On the flow between two co-axial rotating disks. Proc Cam Phil Soc 49:333-341 
6. Brady JF, Durlofsky L (1987) On rotating disk flow. J Fluid Mech 175:363-394

7. Zandbergen PJ, Dijkstra D (1987) Von Kármán swirling flows. Ann Rev Fluid Mech 19:465-491

8. Holodniok M, Kubicek M, Havacek V (1981) Computation of the flow between two co-axial rotating disks: multiplicity of steady state solutions. J Fluid Mech 108:227-240

9. Keller HB, Szeto RKH (1980) Calculation of flows between rotating disks. In: Glowinski R, Lions JL (eds) Computing methods in applied sciences and engineering, pp 51-61

10. Hewitt RE, Duck PW (2000) Non-axisymmetric rotating-disk flows: nonlinear travelling-wave states (herein referred to as HD.). J Fluid Mech 413:287-316

11. Hall P, Balakumar P, Papageorgiou D (1992) On a class of unsteady three-dimensional Navier-Stokes solutions relevant to rotating disc flows: threshold amplitudes and finite-time singularities. J Fluid Mech 238:297-323

12. Hewitt RE, Duck PW, Foster MR (1999) Steady boundary-layer solutions for a swirling stratified fluid in a rotating cone. J Fluid Mech 384:339-374

13. Stuart JT (1960) On the nonlinear mechanics of wave disturbances in stable and unstable parallel flows. J Fluid Mech 9:353-370

14. Hewitt RE, Hazel AL (2007) Mid-plane symmetry breaking in the flow between two counter-rotating disks. J Eng Math 57(3):273288

15. Nore C, Witkowski LM, Foucault E, Pécheux J, Daube O, Le Quéré P (2006) Competition between axisymmetric and three-dimensional patterns between exactly counter-rotating disks. Phys Fluids 18:054102 